\title{
Peer-mentors Reflect on the Benefits of Mentoring: An Autoethography
}

\author{
Sarah Booth \\ Edith Cowan University, \\ Mt Lawley, WA, Australia \\ s.booth@ecu.edu.au
}

\author{
Margaret Merga \\ Murdoch University, \\ Murdoch, WA, Australia
}

M.Merga@murdoch.edu.au

\author{
Saiyidi Mat Roni \\ Accounting Research Institute \\ Universiti Teknologi MARA, Malaysia
}

saiyidi@yahoo.com

\begin{abstract}
Many PhD candidates bring with them a wealth of knowledge and skills; however, these may not sufficiently prepare candidates to work with high autonomy on a project with often limited interaction with the wider research community. A peer-mentor program model, in which a mentor delivers dyadic and group support to higher degree by research students from different disciplines and backgrounds, has the potential to enhance candidates' knowledge and skills. However, the mentors themselves can experience significant advantages, as peer-mentoring can also have a positive effect on the mentors' research experience. In order to further understanding of the potential benefits of peer-mentoring for mentors, three researchers explore their experiences as peermentors through an autoethnographic framework. Through discussing their personal experiences as peer-mentors, the researchers identified a range of benefits for themselves. These benefits involved finding that peer- mentoring enhanced their own learning, fostered reflective practice, and provided current tertiary teaching and research support experience. Peer mentoring also gave them broad exposure to a breadth of disciplines, theories, and methods; provided project management insights; created opportunities for professional networking; supported their social needs; and gave them invaluable insight into other candidate/supervisor relationships. Their role in a peer-mentor model has shaped their experiences as $\mathrm{PhD}$ candidates and also informed their decisions after graduation.
\end{abstract}

(CC BY-NC 4.0) This article is licensed to you under a Creative Commons AttributionNonCommercial 4.0 International License. When you copy and redistribute this paper in full or in part, you need to provide proper attribution to it to ensure that others can later locate this work (and to ensure that others do not accuse you of plagiarism). You may (and we encourage you to) adapt, remix, transform, and build upon the material for any non-commercial purposes. This license does not permit you to use this material for commercial purposes.
Keywords: autoethnography, peer-mentor, reflective practice, teaching as learning, HDR experience

\section{Introduction}

While there have been many studies which explore the benefits of mentoring for Higher Degree by Research (HDR) students, their benefits have been explained with a focus on the mentee (Brill, Balcanoff, Land, Gogarty, \& Turner, 2014; Devenish et al., 2009; Holley \& Caldwell, 2012; Pyhalto, Toom, 
Stubb, \& Lonka, 2012). This paper however, will focus on the postgraduate student as mentor and the benefits of this role to their studies and career paths. To do so this paper draws on the experience of three past and present peer-mentors at Edith Cowan University involved in a model designed specifically for peer support in HDRs including honours and university staff.

While this Edith Cowan University SOAR Centre model has been admired within the tertiary culture to the extent that it has subsequently formed the basis of centres in other Australian universities, such as Victoria University and the University of Wollongong, there has been a paucity of research exploring the SOAR Centre model and its impact on clientele and mentors.

This is particularly relevant as high attrition rates within Higher Degrees by Research (HDR) is a major problem in Australia and many other countries including the UK, United States, Western Europe and Canada (Humphrey \& Simpson, 2012). Within the Australian higher education sector this is of particular concern as, funding is dependent on "student progress and completion" (Devenish et al., 2009, p. 52). This focus on outputs has also brought into question the quality of the work produced.

While graduate education has been traditionally designed as a preparatory program of socialisation for an academic career (Austin, 2002), the need for tertiary institutions to produce HDR graduates who are ready to face contemporary challenges in both academia and industry are increasingly a focus point for institutional reform in graduate training. Aligned with this need to develop a strong and supportive research training agenda (Merga, 2015; Yates, 2010), peer mentors can offer a much-needed service. Easy access to a peer-mentor can relieve the supervisors of some training pressures by providing an alternative avenue for advice and support. This process also allows for the mentors to develop a skill set in advising which can be utilised if they become a supervisor themselves or take leadership roles in the future.

Peer-mentors in the context of this study are ideally high-achieving candidates at the later stages of their PhD or Masters by Research (MBR). They may have developed expertise in a diverse range of areas prior to undertaking their HDR candidature, such as in writing skills or quantitative methods, and therefore have valuable skills to offer. They are also uniquely situated to understand what students are experiencing and the stresses they face, as they themselves are on a similar journey, grappling with similar resourcing constraints and supervisory relationships. While access to peer-mentoring may offer benefits for clientele, this paper focuses on the benefits for peer mentors through exploring the reflections of the previous and current peer mentors who author this paper. This paper examines our reflections of our experience as [Peer-Support Service] Staff and Mentors, using first person pronouns as appropriate to our method when discussing our collective experiences (Humphreys, 2005), and how our time spent in this role affected our success both within our candidature and post-candidature. We will discuss the question: How can an autoethnographical approach explain the benefits of peer mentoring for the mentors?

\section{Methodology}

\section{Autoethnography}

The purpose of this study was to gain insight into our role as a peer-mentor and the impact it had on our studies and career development. As each of our experiences was unique and we each brought with us our own cultural understandings and perspectives an autoethnographical study was appropriate. This aligns with Ellis, Adams, and Bochner's (2011) explanation of the role of autoethnography. They describe the methodology as both a process and a product and one which highlights the complex relationship of the researchers with texts and their audience. This process of drawing on a personal narrative encourages people to make sense of themselves and others and 
offers "... a way of giving voice to personal experience to advance sociological understanding" (Wall, 2008, p. 39).

The methodology itself was developed in the 1980s in response to authoritative manner in which much research had been undertaken, which did not take into account the cultural and social influences of the researcher on their interpretations (Ellis et al., 2011). The autoethnography, therefore, gave researchers a tool in which they could explore personal experiences to give insight into views and experiences different to that of the readers (Ellis et al., 2011). The reflective nature of the methodology also gave benefits to the researcher.

This reflective process aided the transformative potential of the autoethnography; this suggests that by analysing our own experiences we can then shape how we respond to future situations (Struthers, 2014). Furthermore, this interrogation aspect encouraged us to broaden our understanding of our values in relation to others (Starr, 2010). Thus, by understanding our individual context we were better able to locate ourselves within our own history and culture.

The advantage of an autoethnographical study is the insight it offers into our unique perspectives of which some aspects may relate to others. If we can see the benefits of our role as mentor throughout our study and career perhaps others might too.

Although we have limited space to explore in-depth our background, it is important to situate our HDR experience with each of our individual contexts. In this case we have developed Figure 1 to show where we started in the HDR journey and where it has taken us.

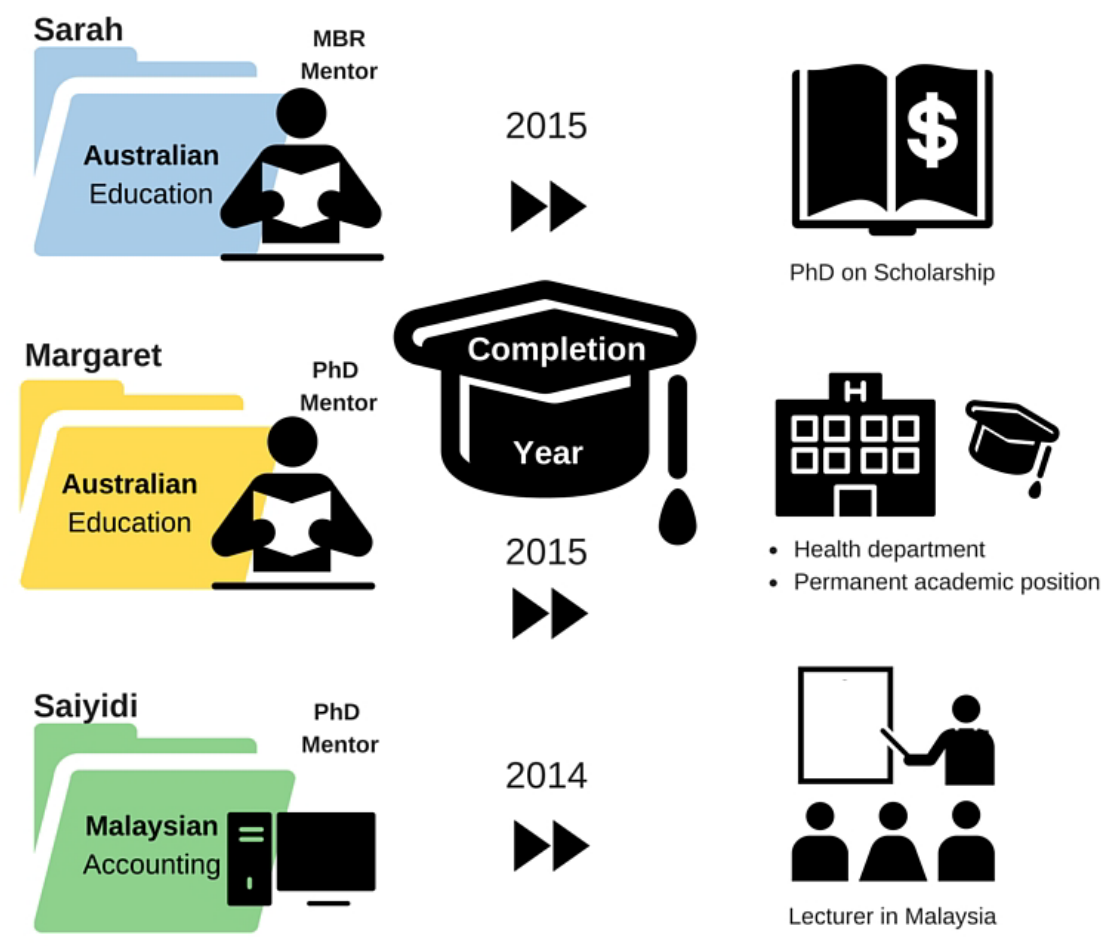

Figure 1: Post HDR completion trajectory of Sarah, Margaret and Saiyidi

All three of us reflected on our experience as peer mentors in the SOAR Centre at Edith Cowan University and discussed our experiences in person, over the phone, through Skype, and via 
email, in order to stimulate critical reflection. As there is currently a paucity of research generated by peer-mentors that can offer insight into our unique situated knowledges (Buzard, 2003, and as such, our purposeful autoethnographic reflection can speak to the validity of allocating resources to provide peer-mentoring as a key component of HDR student services.

The process we took in developing our method was first to discuss our experiences of our time at the SOAR Centre in a free flowing manner and then agree on themes which had become exposed. These aspects we developed into the subsections of the main part of the paper. However, we wrote our responses to the heading in isolation to focus on our individual experiences. Although we had similar themes in our responses it was clear that each had impacted us slightly differently based on our cultural background and prior experiences. The autoethnographical process, in this regard, highlights are individuality as every $\mathrm{PhD}$ candidate brings with them "varying goals, expectations, career histories and family and community responsibilities to their candidature" (Pearson, Cumming, Evans, Macauley, \& Ryland, 2011, p. 538). This links with the understanding that, "When researchers do autoethnography, they retrospectively and selectively write about epiphanies that stem from, or are made possible by, being part of a culture and/or by possessing a particular cultural identity" (Ellis et al., 2011, p. 8). Although both Sarah and Margaret came from education, their research areas were vastly different, and Saiyidi offered his experience as an international student in accounting. To bring our experiences together, the overarching question we asked ourselves was, how did our experience as a mentor shape our study and career path?

\section{Literature and Findings}

\section{Peer-mentoring}

To understand how the role of SOAR has benefited us it is first important to know what the role entailed. The SOAR Centre and its HDR candidate Ambassadors offer assistance not only for HDR candidates, but also for supporting staff. Established in late 2009, this peer-to- peer service consists of ten Ambassadors over two campuses that are selected for their ability to provide a diverse body of skills, knowledge, and expertise. They work six hours a week, which meets the employment limitations for prestigious Australian scholarships such as the Australian Postgraduate Award (APA). SOAR Ambassadors provide dyadic appointments to assist with problems specific to a candidate's research, along with group SOAR seminar sessions on a variety of topics related to the research journey. While performing the role is centrally concerned with knowledge and skill support, in practice SOAR Ambassadors also provide a large degree of social support for clientele. While the Edith Cowan University SOAR Centre model has been admired within the tertiary culture to the extent that it has subsequently formed the basis of centres in other Australian universities, such as Victoria University and the University of Wollongong, there has been a paucity of research exploring the SOAR Centre model and its impact on clientele and mentors.

As per Figure 1, all three authors were employed as SOAR Ambassadors and then promoted to the role of SOAR Mentor. The SOAR Mentor performs the same role as a SOAR Ambassador, though they also have additional team leadership and administrative responsibilities. Hereafter SOAR Ambassadors and Mentors will be referred to collectively as SOAR Staff to prevent confusion, except for in instances where the distinction between the roles is relevant. Two of the authors have now completed their Doctorates; Margaret is an early career academic, while Saiyidi has resumed work at a university in Malaysia lecturing in Education Management. Their reflection is retrospective. In contrast, after completion of her Masters, Sarah continued her SOAR role as a $\mathrm{PhD}$ candidate.

Since Margaret and Saiyidi moved into their early career roles, and Sarah moved into her doctoral candidacy, we have become increasingly aware of the advantages and growth we experienced in 
our peer mentoring roles. We acknowledge that undertaking the peer-mentor role has played a key part in developing our skills to thrive as both masters by research and doctoral candidates to enable a timely completion, along with publications during candidature and preparation to be an early career researcher. What made this role so valuable was the opportunity to enhance teaching and learning skills, foster reflective practice, broaden our research knowledge, and improve skills to discuss our own research and that of others. In addition, the experience exposed us to research processes outside our discipline, increased our professional networking reach and capacity, and enhanced our own and others' social needs during candidature.

Mentoring is recognised as one of the most effective practices in successful student outcomes (Holley \& Caldwell, 2012), however, it is not only the mentee who benefits from the practice. There are considerable positive outcomes for the mentor involved in programs to assist HDR students tackle all that is involved. This for us was the epiphany described by Ellis et al. (2011), which stemmed from our role in the SOAR Centre and highlighted the need to expose the benefits of mentorship for the mentor.

One definition of mentoring is described as "a nurturing process in which a more skilled or experienced person, serving as a role model, teaches, sponsors, encourages, counsels and befriends a less skilled or less experienced person for the purpose of promoting the latter's professional and/or personal development" (Anderson \& Shannon, 1988). This is a leadership skill in and of itself which must be developed. Service and Guess (2015) explain the importance skills of leaders in terms of an emotional quotient which focuses on empathy and also the people quotient which focuses on "connecting and reflecting on the perceptions of others and moving from fixation to adaptation" (p.59). As a peer-mentor these are skills which take time but are vital for the mentor to be effective.

One important benefit of peer-mentoring, also found in many forms of mentoring, is reciprocal learning (Holley \& Caldwell, 2012). In our experiences as peer-mentors we found this particularly powerful in our own project development as we were learning from teaching. As we encouraged critical thinking and assisted students in making personal and academic decisions we were also developing these same aspects within ourselves (Brill et al., 2014).

\section{Teaching Enhances Learning}

Teaching enhancing learning was one topic which came up immediately through discussions on our role as mentor. Our learning was enhanced through the practice of developing a particular skill and building on our previous knowledge in order to assist our clientele. This was conducted either through the process of SOAR sessions which were a seminar format or through one-to-one appointments. Through the act of teaching we were deepening our understanding of a particular skill, topic, or software program.

There is a wide body of research in education and psychology that explores the potentially reciprocal relationship between teaching and learning, with conceptual understanding enhanced through the process of sharing with others. For example, expectation of teaching content has been found to improve learning and knowledge organisation. Participants expecting to subsequently teach material acquire a better knowledge of material than participants merely expecting to be tested on it (Nestojko, Bui, Kornell, \& Bjork, 2014). As mentors we have improved our learning through the facilitation of workshops which focus on enhancing the HDR candidate experience. These workshops were often specific, such as improving writing skills or teaching software programs to enhance data analysis, which led to a deeper understanding of concepts and software.

The value of teaching as a learning tool is so broadly recognised that the introductive of virtual "teachable agents", where students learn by teaching a computer, has been flagged as an innovation that can support cognitive retention of meaningful learning (Chin et al., 2010), perhaps due to 
the sense of responsibility the teaching role evokes, even when the teachable agent is non-human (Chase, Chin, Oppezzo, \& Schwartz, 2009). When participants ask us questions within workshops they stimulate our cognitive process as we must think quickly to answer. Reflection is a key factor in teaching as a tool for learning.

Sometimes it is not until we reflect on our own experiences that we see the value of what we have done. The concept of teaching enhancing learning was well understood all three authors, however it still took the reflective process of an autoethnography to understand the deeper impact of particular teaching methods and their effects on us as learners. The SOAR Sessions which were aimed at students from a range of disciplines encouraged us to think widely about a particular application of our skills and knowledge. Each of our accounts highlights the common thread of our learning through teaching but also the different perspective we took.

\section{Margaret}

I found that teaching the seminars forced me to broaden my understanding of key areas in which I already had foundational expertise. As such, I was building my knowledge in areas that were already of interest; therefore I was both intrinsically and extrinsically motivated to acquire further knowledge. As an experienced educator, I was able to recognise that I was experiencing extrinsic motivation, "which refers to doing something because it leads to a separable outcome" (Ryan \& Deci, 2000, p. 55); this arose from my need to provide quality instruction in the area in order to meet my employment requirements and achieve favourable student feedback reports to support my own professional progression and the reputation of the Graduate Research School. However, I also experienced an even greater degree of intrinsic motivation "doing something because it is inherently interesting or enjoyable" (Ryan \& Deci, 2000, p. 55), which arose from my pre-existing keen interest in the areas covered, and my desire to improve my own skills and knowledge. Intrinsic motivation is important as it results in highquality learning and creativity (Ryan \& Deci, 2000).

\section{Sarah}

Preparing for seminars or SOAR sessions meant that I had to go back over material I had learnt previously. This reinforced and highlighted some key concepts of a particular software program and reminded me of uses for it that I had previously forgotten. At the time of writing this article I was still in the SOAR role and by reflecting on these experiences it has enabled me to look for workshops to facilitate in which I would need to learn a new skill to teach and hence increase my skillset.

\section{Saiyidi}

In this respect, it was important I make sufficient preparation to conduct a SOAR session and resourcefulness while attending to enquiries. These brought about transformative learning attitude (Addleman, Nava, Cevallos, Brazo, \& Dixon, 2014) to gather resources in order to explain 'how' and 'why', rather than a description on 'what', to the audience who had to understand the construction of emergent knowledge to be applied in their field of study. The preparatory and delivery processes not only served the clientele but also myself throughout my $\mathrm{PhD}$ candidature.

The one-to-one appointments present a different kind of challenge and a different skill set. For the seminars we could prepare, however, the appointments required an immediate response to questions without necessarily having the luxury to research them beforehand. However, this fluid process of learning with the client encouraged a different set of cognitive skills. We discuss each of our experiences and how they influenced us. 


\section{Sarah}

I found that due to the expectation placed upon me as a source of assistance, I was highly motivated to problem solve quickly. Where issues were beyond my scope of understanding, I would refer the student on as appropriate, building my understanding of the multiple avenues for support within the institution. There were often times when I would work with my peers in finding a solution, I would provide a little information and they could provide the next step. This was particularly the case with software programs. I developed a greater understanding of how various software programs worked simply from being asked to help.

\section{Margaret}

I also found that providing support as a SOAR officer re-energised me, maintaining my intrinsic motivation along my research journey. Even when the research area was far removed from my own area of expertise, the opportunity to support students in relevant generic skills, such as academic writing or thematic coding of qualitative research data, made me aware of the value and transferability of the research skills that I was acquiring through participating in regular institutional research training and through practice, in conducting my research.

\section{Saiyidi}

Prior to the enrolment as a PhD candidate, I taught in a Malaysian university in the field of accounting information system. The teaching experience helped me with pedagogical approach to attend to enquiries from SOAR clientele. While the enquiries were mostly on research technicalities, the pedagogy remains similar. Therefore, I was transferring my skills I had previously learned and adapting them to new situations.

From each of our accounts our learning would not have developed as quickly or to the same extent without the pressure of assisting those who came to us for help. We were able to understand this through the process of reflection. This reflection process was both from this autoethnograpical study and also during our roles as mentors. To prepare for teaching as SOAR Staff each of us had to disseminate our own practice, which highlighted aspects which could be more effective and those which we wished to share.

\section{Fostering Reflective Practice}

Reflective practice not only improved our role as mentors, giving us greater understanding in our work, it also linked closely with our teaching as learning. By analysing our teaching practice we were forced to think of learning through the student perspective by drawing on our roles as both students and mentors. These reflections took place in the form of looking at our own cultural background in relation to international students, comparing and contrasting our research journey with others, and using these insights to reflect on our teaching practice.

As mentors we were in an environment which fostered reflective practice due to our exposure to many of our peers. They would bring with them a wealth of knowledge along with problems and issues more specific to their individual circumstances. Yet, from their experiences both good and bad we could reflect upon our own and learn from them. We could then share these insights to prevent similar calamities and promote effective work habits.

Reflective practice refers to the "the process of thinking about the work we undertake" (Thompson \& Pascal, 2012, p. 319). It transcends the habitual and locks onto our mental capabilities to analyse what we have done in order to learn and improve from it. This analysis of our actions ensures we are aware of what we are doing which prevents actions and habits from going against our values (Thompson \& Pascal, 2012). In our role as a SOAR Staff member this was particularly relevant. It was essential for us to constantly reflect on how we responded to students 
and problem solving. We were also recipients of client feedback, which guided our selfimprovement.

This reflective practice also encouraged understanding of cultural differences within the student cohort, such as the potential different cultural determinants involved in willingness to communicate (McCroskey \& Richmond, 1990).

Furthermore, this greater understanding of cultural differences encouraged a perspective transformation in each of us where those practicing it become more aware of cultural conditionings through "consciousness raising" (Mezirow, 1978, p. 102). The SOAR Centre was heavily utilised by international students, therefore understanding how their cultural conditionings could impact on their understanding of supervisor expectations and general expectations of them was essential in giving appropriate advice and help. We found that when assisting international students our role became varied.

Saiyidi's experience as an international student was enhanced by his role as SOAR Staff. His cultural background meant there were sometimes barriers to overcome his own research in a foreign country and in how he reflected in his role as mentor. International students face many challenges when studying abroad as they adjust to the culture of their host country. Saiyidi was in a unique position as mentor as he was able to reflect on his experiences as an international student to better assist his international peers and understand his circumstances more objectively to help problem solve.

\section{Saiyidi}

The role of a SOAR Staff member not only allowed me to be more intimate with deeplearning of new knowledge, but also opened up an avenue of multi-cultural interaction with domestic and international students. This opportunity enhanced my intercultural competence (Jackson, 2015) through conversing with individuals from diverse linguistic and cultural backgrounds. Such engagement expedited my progression on intercultural development continuum (Hammer, 2012) to ethno relative stage (Hammer, Bennett, \& Wiseman, 2003) where adaptation and integration of my cultural perspective was assimilated to fit the host environment. There were, of course, expectation gaps, which exist between how things were done in my home country and how they were dealt with in Australia. However, the deficits of these expectations, entrenched through cultural lens, were covered by a surplus of good practices, seen and experienced throughout the tenure of assuming the role of SOAR Staff.

For Margaret, international students posed different challenges.

\section{Margaret}

In a number of cases, I found myself fulfilling both a technical role, in supporting oral communicative competence, and providing a socially supportive role in encouraging students to communicate their issues and concerns clearly within their supervisory team. I introduced students to simple techniques such as using MP3 players to record their supervisors, and making their own position known by asking supervisors to slow down and asking them to clarify their expectations. I was surprised to discover that some international students had resigned themselves to exclusion, sitting through supervisory meeting with limited understanding of the discussion around them. This made me aware of the necessity to not take communicative success for granted, even if the student was giving non-verbal or verbal cues indicating comprehension; some students had achieved quite sophisticated mechanisms for feigning comprehension in order to conceal what they perceived as personal failings in communication, taking full responsibility for the success or otherwise in the transfer of ideas and information in their supervisory team. 
Sarah was also surprised at the cultural differences and the impact they had towards international student's attitudes towards the research process.

\section{Sarah}

I became more aware of the impact of the power relationship between students and their supervisors particularly in the case of international students. Until I spoke to international students I had not understood the difficulty many had with asking for more clarification from their supervisors.

International students were just one area where our preconceived ideas had to change to adapt to understand reasons behind challenges students faced. There was also a generational difference in many of the research candidates, which in many respects reproduced similar issues to those from a different culture. Some mature students often struggled with digital literacy, in particular the use of new technologies and software programs needed to explore their research. There was often some reluctance to take up the new technologies despite the advantage it would give the candidate. Through teaching about a research specific software program, Sarah often came across this response.

\section{Margaret}

I was also required to reflect on my assumptions about so-called digital natives, discovering that it could not be assumed that because a person was from a younger generation, that they would necessarily have high competence. In my mid-thirties at the time of my candidature, I found myself in the unexpected position of providing technical support for students in their mid-twenties; with even relatively simple word functions such as "find and replace" in a Word document posing a challenge for some students. I subsequently discovered that this experience was consistent with research challenging the dichotomy of "digital natives" and "digital immigrants" (Bennett, Maton, \& Kervin, 2008; Kennedy, Judd, Churchward, Gray, \& Krause, 2008), which suggests that young Australians are a highly heterogeneous group of technology users (Kennedy et al., 2008).

Understanding our preconceived ideas was part of comparing and contrasting our research journey to that of others. In each of our accounts we bring to light our learning of our preconceived ideas and how this understanding influenced our study and teaching practice.

\section{Saiyidi}

This role also extended the boundary of my comfort zone and propelled my self-confidence to a greater height. One of the most valuable aspects of my experience was the issues and challenges faced by clients at different stages of their $\mathrm{PhD}$ programme. While I provided support and solutions to my clients facing difficulties in stages I had gone through, I also found myself assisting those who were ahead of me. Furthermore, by looking for solutions to clients who, at the time, were ahead of my own $\mathrm{PhD}$ stage, it set my self-confidence trajectory in the right direction. This helped me to expect potential conundrums in the later stage of my study, and I became aware of available initiatives and supports.

\section{Sarah}

Before becoming SOAR Staff I did little reflective practice, this was due to my isolated state and therefore a lack of communication with other candidates. Once I had become Staff I had more opportunities to reflect on my own practice simply from hearing others experiences. I could then ask myself probing questions about my work practices and emotional responses to feedback. It also prepared me for potential difficulties such as issues with finding examiners 
and handling feedback. I became more informed about the entire process of completing an M.Ed instead of focusing only on my stage of candidature at the time.

By understanding our journey in relation to others we were able to gain insight into our teaching practice and how to improve it.

\section{Margaret}

The SOAR role forced me to constantly reflect on my research journey and my learning; every time I acquired a new skill, I found myself envisioning how to translate it into teachable content and learning experiences with cross-disciplinary relevance. Introspection and reflective practice was therefore an essential skill in undertaking my role as SOAR Staff effectively.

\section{Sarah}

I often spoke to peers who were reluctant to learn a new program as they believed it would take too much time away from their work. Explaining the benefits of learning the technology such as NVivo for qualitative research analysis, reinforced the importance of such programs for my own research. Learning to explain the program in a way that would be understood by someone not familiar with complex programs also enabled me to reflect on the aspects of the program that were most useful.

As such, constant reflection challenged our assumptions and forced us to engage in an iterative process of shaping the learning experiences that we provided, as well as increasing our capacity to be both flexible and responsive.

By fostering reflective practice within our role as mentors we were better equipped to face the challenges of academia through vocalising our research.

\section{Current Tertiary Teaching and Research Support Experience}

While gaining tertiary teaching and research support experience is beneficial when viewing doctoral candidature as an academic apprenticeship intended to prepare participants for early career research, it also enabled us to improve our ability to talk about our research and the research of others. We found we were constantly interacting with candidates outside of our disciplines. This was both challenging and valuable as it enabled us to understand a wider range of processes and research frameworks. It also forced us to communicate our research in Plain English, not overladen with discipline-specific jargon, a transferrable skill with value beyond academia.

With jobs in academia across the globe increasingly scarce (Altbach, 2015), gaining a broad range of skills is essential for paid employment after a doctorate. This role prepared us for jobs within academia from our teaching experience as SOAR Staff members and outside of academia, as a result of our wide range of learning and skills. For those SOAR Staff from disciplines other than education, this tertiary teaching opportunity provided scope to ascertain if teaching at university would be a desirable profession that is compatible with the interests and skills of the SOAR Staff.

In this regard, when the tertiary environment provided additional opportunities for exposure to a breadth of discipline, theory, and method that extends beyond the capacities of the supervisory team, students can be exposed to a greater range of research lenses and opportunities (Green, 2005). To a lesser extent, our clients are also being introduced to a wider variety of research areas through their interactions with us.

During workshops we also needed to cater for a wide range of disciplines which ensured our workshops were broad in scope. Our workshops would often involve a discussion component where participants could share their research and experiences. This added to our knowledge on 
how a variety of researchers problem solved which we could then use later for our own research or assisting other students. This was particularly evident for Margaret as her role as an early career academic drew upon many of the skills she gained through her mentor position.

\section{Margaret}

My research support experience has been invaluable in my role as an early career academic. In my first year out, I have a PhD student and three Masters students. I find myself continually drawing on the research skills and knowledge that I acquired in my SOAR Staff role to perform my supervisory role effectively. Being a peer mentor has made me very respectful of the need to determine my students' unique research training needs on an individual basis. I closely consider how to best deliver support in order to optimise progressive student autonomy, and also I endeavour to reduce my students' sense of isolation by creating collaborative forums and opportunities for "team" meetings, as I work at a university without a current cross disciplinary peer-mentoring model for HDR student support.

\section{Sarah}

Although at the time of this article I was still completing my candidature, my role as SOAR Staff gave me the confidence to seek opportunities for employment within the university. These opportunities often arose from university staff becoming aware of my skills through sharing office space. An example of one such incident was a workshop on the qualitative software program NVivo; I became the facilitator after offering to assist in the workshop. I have also facilitated writing weekends for HDR students and staff members. This has increased my confidence in my knowledge and skills to improve the HDR experience.

These experiences have not only enabled each of us to draw on what we have learnt in our role as SOAR Staff and implement it in our current endeavours, but it has also broadened our understandings of many disciplines, theories and methods.

The collaborative environment was, therefore, a valuable space for us to expand our knowledge and consolidate existing theoretical frameworks. It also enabled us to foster working relationships post-doctorate. This paper is one such collaboration that came about because of our awareness of the current gap in understanding of the benefits of the peer support model for mentors and our general advocacy of this model due to our positive experiences as SOAR Staff. All these experiences helped to develop a generic set of skills to participate in the workforce after completion (Pearson et al., 2011).

Our understanding of the importance of professional networking became clearer during our employment at the SOAR Centre. Not only was our role a natural form of networking through clientele but also amongst staff. While we did not publish or conduct research together while working in our SOAR roles, we did feel very much a part of a team. Despite Margaret moving to another institution, and Saiyidi returning to Malaysia, we retained our relationship with each other as former SOAR colleagues, who we viewed as valuable peers. As aforementioned, the HDR candi-

dature journey can be very isolating, which is significant for psychosocial reasons, but also as it is a somewhat unrealistic experience, as much of the high-level research conducted in tertiary institutions is highly collaborative.

\section{Project Management Insights}

Project management is an aspect of the research journey that is likely to be underestimated. Each of us was exposed to a variety of ways students managed or did not manage their candidature. However, the role of SOAR Staff could take time away from our own projects if we were not careful, through the manipulation of students needing our help or as a form of procrastination 
from our own view point. The importance of project management was therefore a factor that we learned not to underestimate if we were to finish on time. This aspect of the HDR journey is highlighted by Gorringe and Hotchman (2006) who state that the need for timely completion and growing accountability for research quality underpin the increasing emphasis on effective project management during HDR candidacy. As SOAR Staff we played a key role in supporting students' project management, while at the same time, managing what was in many cases the most significant funded project we have ever conducted. Margaret discusses how important it was to understand her limitations and strengths in this process.

\section{Margaret}

In my SOAR role, I became aware of the strengths and limitations of various project designs, as well as the wide variety of emphasis on careful and rigorous project management between individuals, supervisory teams and disciplines. I watched a number of high-risk projects stall or fail due to lack of consideration of mitigating factors, which made me much more cautious in my own approach. I became aware of the importance that a high level of planning be accompanied with willingness for flexibility within the constraints of ethics and faithfulness to the original research purpose.

As illustrated by Margaret, an understanding of factors which enables peers to succeed or fail was invaluable to improving project management. Sarah approached project management through observing both positive and negative habits of peers.

\section{Sarah}

A combination of completing a Masters in Education (MEd) and working at the SOAR Centre enabled me to better project manage my $\mathrm{PhD}$. I was also aware of the challenges involved in balancing part-time work and study. Developing effective work habits is essential for quality of work and enhancing the probability of a more enjoyable research experience (Devi, Sunita $\&$ George, 2012). To develop my own positive habits I needed to dedicate time to write and the accountability of working with other people. Producing regular writing output was central to my approach, which was informed by Kearns and Gardiner's (2012) emphasis on the importance of writing with high frequency from the early stages of candidature. As this issue also had currency for other students across disciplines, I decided to develop a specific writing time for students. This developed into a weekly writing session of three hours for students needing the same accountability as myself. Furthermore, this culminated in a full day of writing and subsequently a writing weekend, which has since been replicated. These sessions have often helped both myself and those involved to establish or re-establish efficient writing habits, leading to improved research outputs.

Saiyidi explored the difficulty of managing the competing requirements of a $\mathrm{PhD}$ and work.

\section{Saiyidi}

Being a SOAR Staff member at the same time as a $\mathrm{PhD}$ student helped me to identify possible prospects to streamline two apparently competing output requirements. Attending the enquiries and conducting SOAR workshops were some of the main tasks in my role at SOAR, while the progression of my thesis was my major output in my own study. In order to align these outputs, I planned my SOAR workshops to be paralleled with my thesis progress. For example, while I was at the data analysis stage, I conducted SOAR workshops on data analysis. The alignment of these outputs not only benefited the clients but also strengthened my approach to data analysis for my thesis. 
This process of reflecting on the projects of our peers alongside our own has enabled us to see the value of our position as SOAR Staff. The process of collaboration through sharing resources and practices has also led to a culture of networking within the role.

\section{Social Needs}

Our social needs were more likely to be met as SOAR Staff than as simply a HDR candidate. We were also in a position to encourage others to socialise more through coming to SOAR Sessions and also developed friendships. This was particularly important as doctoral candidacy can be a period characterised by social isolation, and research suggests that this may be a significant factor in attrition (Ali \& Kohun, 2006; van de Schoot, Yerkes, Mouw, \& Sonneveld, 2013). As such, efforts to mitigate social isolation may not only be of significant individual benefit, they may also enhance institutional completion rates. As SOAR Staff, we not only help to mitigate social isolation in our clients that we work with, the process also forces us into one day a week of extroversion. This was beneficial in breaking the habit of working alone which many HDR students fall into. Margaret explains this benefit.

\section{Margaret}

I found myself eagerly looking forward to the opportunity to think about someone else's research, a journey in which I was not so self-invested. I looked forward to the interpersonal interaction and the sense of reward I felt in helping other move forward who were experiencing similar challenges.

Sarah had a particularly isolating experience through much of her M.Ed. However, this changed dramatically once she became a mentor.

\section{Sarah}

Before becoming SOAR Staff I was very isolated and worked slowly and sporadically; I rarely spoke to other HDR students and became entrenched in my own research. As a result, all my reading and inquiry was self-focused. Once I became SOAR Staff I began to encounter and absorb an abundance of ideas. I became engrossed in extrapolating how others approached their research. It also allowed me to gain insight into areas of research I was interested in, yet in order to focus on one area of study, I could not pursue at that time.

The forced socialisation also benefited Saiyidi. For international students to gain the full benefits of studying overseas socialisation is imperative. Despite this, "Only a small percentage of international students reported having close friendships with domestic students, however, this was due to lack of opportunity and/or preference for friendships with co-nationals" (Andrade, 2006, p.134). Saiyidi's role as [Peer-Support] Staff gave him an advantage through his forced interactions with both domestic and international students.

\section{Saiyidi}

Taking up the role of SOAR Staff not only shaped my professional interaction but also positively affected my socialisation. I became more receptive on others' needs and the way their perspectives were moulded by engrained culture of their homeland. This was reflected in my own cultural background allowing me to respond in ways that were acceptable in the current environment. My socialisation was not only limited to in-person interactions but also extended through an online medium through a good access to advanced hardware, applications and information technology infrastructure.

As social relationships are a key factor in contributing to a successful completion (Littlefield, Taddei, \& Radosh, 2015), discussing issues with peers was extremely helpful. Also my 
knowledge of each of the SOAR Staff skills enabled me to know who to talk to for a specific issue. Therefore, this role enabled me to efficiently source information from knowledgeable peers and also to feel comfortable asking my colleagues for assistance. My role was often an exchange of knowledge and ideas, fostering collegiality.

As the SOAR office was open during the week it encouraged SOAR Staff to engage socially. This was particularly helpful when deadlines were looming, providing valuable stress relief which enabled re-focussing; research supports the contention that socialisation relieves stress (Littlefield et al., 2015). As a result, developing relationships in the academic arena can help relieve stress and anxiety and at the same time provide academic support. A source of stress that is common amongst HDR students is the relationship with the candidate/supervisor relationship.

\section{Insight into Other Candidate/Supervisor Relationships}

Doctoral candidates are often intrigued by other candidate/supervisor norms as they negotiate their own, sometimes-fraught relationships. As SOAR Staff we were positioned to learn much about the dynamics operating in the teams of other students, knowledge that could then be applied to our own contexts. In our role we are in a unique position, as a supportive peer, to hear what many students experience in the candidate/supervisor relationship, which varied on a long continuum from highly supportive to destructive. The supervisory relationship is crucial in a successful completion, particularly due to the aforementioned increasing emphasis on timely completion (Adkins, 2009; Ives \& Rowley, 2005). There are, however, many factors that can negatively impact on the relationship candidates have with their supervisor, such as the maintenance of appropriate boundaries, as the development of friendships can cause issues insofar as upholding professionalism (Hemer, 2012). This issue could also apply to SOAR Staff and their professional relationship with their peers, including the issue of mentoring friends. The relaxed atmosphere of the SOAR Centre and the low responsibility of the Staff make it easy for these boundaries to be surpassed. This was particularly the case for Margaret where her generosity was abused by a student.

\section{Margaret}

I found myself having to re-establish boundaries with some students who became excessively dependent on my writing support, to the extent that I felt that I was bordering collusion due to the volume of my contributions. I tried to increasingly foster student confidence and gradually withdraw support. There was one student who would visit me, take advice from me and then turn around and use it as an excuse for not doing anything, using the argument that my advice and his supervisor's advice differed. This is why we needed to consistently state that our advice should always be seen as secondary to the supervisor's advice. I found working with this student really frustrating, as he was very manipulative and, as a peer, I was not in a position of power or real influence; I felt like a pawn in his greater game of procrastination. Fortunately we had a lot of support from the Project Manager who was in charge of managing the SOAR team, so we always had someone that we could seek guidance from when issues arose.

These unbalanced encounters with students was one aspect of the role of SOAR Staff which was not always easy to deal with. Often the instigation of these issues was the breakdown of the supervisor student relationship. Although this could sometimes place the SOAR Staff members in a difficult position, they were also in a position to help. The mentor could give an objective opinion about a situation which may be highly emotional for the student. Sarah experienced a situation where a student felt abandoned by her supervisor.

\section{Sarah}

A student came to me in a highly emotional state as her supervisor had left for long service leave and she had no way of contacting the supervisor for reassurance. By having the oppor- 
tunity to voice her fears and explain her grievances she was able to reflect upon her situation and better understand it.

I also experienced issues with students becoming dependent, especially at crucial junctures in their candidature. In these high-stress, high-accountability periods students often asked more of me than I felt comfortable to supply, such as a request to edit or proof-read their entire thesis. Ethical concerns were subsequently raised and I gave advice without taking on their work. I also found that at these times what students were really looking for was validation that they were on the correct path, something which some students felt they were not receiving from their supervisors.

The exposure to these difficulties encouraged us to reflect on our relationships with our own supervisors and helped us to understand the significant factors in the relationship. For Margaret, who at the time of this article works in academia as an associate supervisor, this was highly informative. At the same time, realistic expectations were fostered, highlighting concerns if their supervisors were not performing as they should. Being able to discuss these dynamics with SOAR Staff enables students to understand if they need to change supervisors or if the problem can be solved with a meaningful conversation and a new supervisor agreement plan; however, the SOAR Staff could not make this decision for them and tended to refer them as soon as practicable to the appropriate support team. The PhD program is unique in that students need a tailored program for their studies. Furthermore, each student embarks upon the doctoral journey with a unique set of needs and experiences (Moxham, Dwyer, \& Reid-Searl, 2013), which is also true of Masters by Research students. In this regard finding a supervisor that fits is essential for a successful completion.

Saiyidi noticed that there was an expectation gap existed between candidates and the supervisors. While the supervisors envisage guiding the candidates toward higher level thinking, on certain occasions, the candidates anticipated full supervisory supports from research fundamentals to analytical emphases. These gaps were addressed by providing assistance for the candidates to work on their tasks while at the same time emphasising on the philosophical nature of supervisorcandidate role.

\section{Discussion and Conclusion}

The difficulties involved in undergoing a PhD or any Higher Degree by Research are well-known (Adkins, 2009; Ives \& Rowley, 2005). Candidates must juggle family life along with learning new skills and building a good relationship with their supervisor. As SOAR Staff, we have been in the midst of these challenges ourselves. Through the process of this autoethnographical study we have reflected upon our practice as SOAR Staff and how this has influenced our current positions in our respective institutions. Looking back at the work we did and the insights we have gained has brought greater clarity to understanding how our roles as SOAR Staff have positively impacted our overall HDR experience.

To be a successful HDR candidate we hope to develop our research skills and knowledge beyond our discipline. In order to achieve this, we must move away from the confined world of our discipline and develop transferable skills. Through our role as SOAR we have begun to understand the importance of reflecting and asking questions of ourselves rather than seeking answers from others. We have also understood the importance of interaction with peers and relating to their cultural backgrounds in order to assist them. We have also understood the importance of not becoming a crutch for students but to develop skills which increases both ours and their learning independence.

Through our role at the SOAR Centre we have still been tied to our research and thesis writing. There were times when it was easier to assist a client rather than work on our theses; however, 
this danger was often negated by the support we knew was on offer. Discussion about project management with other SOAR Staff and students occurred often. This support in an area which is easily overlooked by many students was significant to our completion.

With attrition rates a consistent problem, universities need to look for ways in which to support HDR candidates. This SOAR has enabled us as mentors to improve our experience and also others around us by developing a culture of sharing information and services.

As our role as SOAR Staff involved teaching peers, it has enabled us to reflect on our own practice in order to teach the material to a high standard, and for it to be comprehensible to those outside of our disciplines (Thompson \& Pascal, 2012). This form of reflective practice has cemented difficult concepts and improved our understandings of them (Nestojko et al., 2014). This practice of teaching to participants outside of our discipline increased our understanding of knowledge translation, a vital tool in communicating research.

The small group sessions constitute the first teaching experience for some SOAR Staff, giving them the opportunity to decide if lecturing or tutoring is a viable career option. This opportunity to understand our skillsets and discover where we were lacking was valuable when considering capabilities. With access to peers with a wide range of skills it became easy for us to seek answers from them and solve issues sooner.

The relationship between student and supervisor is crucial to candidate success, and as SOAR Staff we became acquainted with the wide range of potential variations in its manifestation across the university. The subsequent knowledge we acquired in how to effectively manage this relationship is also something we have been able to share with clients, to let them know that many other people have difficulties which can be solved. This may be due to cultural differences, where students may perceive a greater power imbalance than domestic students, which may obstruct their ability to learn (McClure, 2007). As a result, our presence has enabled students to problem solve issues in the supervisory team with peers with whom they feel comfortable. This is particularly important as the relationship a candidate has with supervisors significantly impacts a timely completion (Moxam et al., 2013).

We were also able to assist supervisors and students in specific research skills. This was particularly the case with computer software programs, as many of the SOAR Staff had greater expertise than many supervisors. These skills took pressure off supervisors and gave a greater opportunity for students to access. It also benefitted us the mentors as we were able to hone our skills and add more skills to enable our greater chance at finding employment. This was particularly evident with Margaret who found employment immediately after graduation and later found an academic position. Although this was largely due to well-developed skills and publications, the role as SOAR enhanced these skills and gave her the opportunity to share them.

Our role as peer-mentor also enabled us to have a deep understanding of the pressures of project management as we going through the same process. Despite the process differing for each candidate, as they brought with them their own challenges and needs (Mason, Goulden \& Frasch, 2009), we were able to delve into our pool of knowledge to source solutions. The ability for clients to contact both in person and through phone and email, SOAR Staff with a range of expertise ensured ready access to an appropriate support.

The availability of SOAR Staff was an important factor in helping lessen the effects of isolation in many $\mathrm{PhD}$ candidates (van de Schoot et al., 2013). We were also the first point of contact in many cases for many concerns; this was particularly the case for students who did not want to ask what they felt were 'stupid' questions of their supervisors. In addition, the role lessened the feeling of isolation amongst us as SOAR Staff. Through interactions amongst ourselves and other professional and academic staff we were able to develop our professional circle. This particular 
insight has brought into focus the importance of networking, not just for socialising but developing professional contacts.

Conversely, as indicated, the peer relationship was not without its challenges, and as SOAR Staff we needed to ensure that we clearly communicated our position of influence as being secondary to that of the client's supervisory team. We also needed to actively avoid over-dependence, which was somewhat difficult to negotiate due to the fact that clients were also peers, and often extant or subsequent friends. The blurring of the personal and professional posed an issue; however, the top-down management support that SOAR Staff received enabled us to seek assistance in negotiating these difficulties as required. Professional relationships also became friendships amongst SOAR Staff, which may be of particular significance in giving international SOAR Staff a sense of belonging and place.

Through the reflection process of this autoethnographic study of three SOAR Staff mentors, Sarah, Margaret and Saiyidi, we have discussed the challenges of conducting a HDR and how the peer-mentor role has helped to develop ourselves as researchers. Furthermore, it allowed us to implement preventative measures to tackle the variety of difficulties encountered during candidature. This position as a member of staff developed our identity as researchers and encouraged us to become more active within the research community.

These benefits, although mostly described from our perspective as mentors, have also clearly assisted those who have used the services offered. In addition, they have clearly contributed to a broadening of our possibilities beyond the period of candidature. This paper seeks to contribute to discussion around higher education reform, placing the potential of a peer-support model as an advantageous adjunct that can be easily incorporated into existing training models. Further research exploring the impact of peer-support on client experiences, comparison of the efficacy of different peer-support models, and the features of peer-support models that offer the greatest benefits for students, mentors, supervisors and their institutions is clearly indicated, so that the full potential of this model can be realised, and so that its widespread adoption can be advanced.

\section{References}

Addleman, R. A., Nava, R. C., Cevallos, T., Brazo, C. J., \& Dixon, K. (2014). Preparing teacher candidates to serve students from diverse backgrounds: Triggering transformative learning through short-term cultural immersion. International Journal of Intercultural Relations, 43, 189-200.

http://doi.org/10.1016/j.ijintrel.2014.08.005

Adkins, B. (2009). PhD pedagogy and the changing knowledge landscapes of universities. Higher Education Research \& Development, 28(2), 165-177.

Ali, A., \& Kohun, F. (2006). Dealing with isolation feelings at IS doctoral programs. International Journal of Doctoral Studies, 1, 21-33. Retrieved from http://www.informingscience.org/Publications/58

Altbach, P. (2015). The deteriorating guru: The crisis of the professoriate. International Higher Education, $36,1-2$.

Andrade, M. S. (2006). International students in English-speaking universities: Adjustment factors. Journal of Research in International Education, 5(2), 131-154. doi:10.1177/1475240906065589

Austin, A. E. (2002). Preparing the next generation of faculty: Graduate school as socialization to the academic career. The Journal of Higher Education, 73(1), 94-122.

Bennett, S., Maton, K., \& Kervin, L. (2008). The 'digital natives' debate: A critical review of the evidence. British Journal of Educational Technology, 39(5), 775-786.

Brill, J. L., Balcanoff, K. K., Land, D., Gogarty, M. M., \& Turner, F. (2014). Best practices in doctoral retention: Mentoring. Higher Learning Research Communications, 4(2), 26.

Buzard, J. (2003). On auto-ethnographic authority. The Yale Journal of Criticism, 16(1), 61-91. 
Chase, C. C., Chin, D. B., Oppezzo, M. A., \& Schwartz, D. L. (2009). Teachable agents and the protégé effect: Increasing the effort towards learning. Journal of Science Education and Technology, 18(4), 334-352.

Chin, D. B., Dohmen, I. M., Cheng, B. H., Oppezzo, M. A., Chase, C. C., \& Schwartz, D. L. (2010). Preparing students for future learning with teachable agents. Educational Technology Research and Development, 58(6), 649-669.

Devenish, R., Dyer, S., Jefferson, T., Lord, L., van Leeuwen, S., \& Fazakerley, V. (2009). Peer to peer support: The disappearing work in the doctoral student experience. Higher Education Research \& Development, 28(1), 59-70. doi:10.1080/07294360802444362

Devenish, R., Dyer, S., Jefferson, T., Lord, L., van Leeuwen, S., \& Fazakerley, V. (2009). Peer to peer support: The disappearing work in the doctoral student experience. Higher Education Research \& Development, 28(1), 59-70.

Devi, V. R., Sunita, V., \& George, M. (2012). Effective work habits for professional growth. IUP Journal of Soft Skills, 6(3), 38.

Ellis, C., Adams, T. E, \& Bochner, A. P. (2011). Autoethnography: An overview. Historical Social Research/Historische Sozialforschung, 12(1), 273-290.

Gorringe, M., \& Hochman, M. (2006). The complexities of managing research projects: An ongoing study of developing a quality framework and measuring perceptions of service quality at UniSA. Journal of Research Administration, 37(1-2), 113.

Green, B. (2005). Unfinished business: Subjectivity and supervision. Higher Education Research \& Development, 24(2), 151-163. doi:10.1080/07294360500062953

Hammer, M. (2012). The intercultural development inventory: A new frontier in assessment and development of intercultural competence. In M. B. Vande, R. M. Paige, \& K. H. Lou (Eds.), Student learning abroad (pp. 115-136). Sterling, VA: Stylus Publishing.

Hammer, M., Bennett, M., \& Wiseman, R. (2003). Measuring intercultural sensitivity: The intercultural development inventory. International Journal of Intercultural Relations, 27, 421-443.

Hemer, S. R. (2012). Informality, power and relationships in postgraduate supervision: Supervising PhD candidates over coffee. Higher Education Research \& Development, 31(6), 827-839.

Holley, K. A., \& Caldwell, M. L. (2012). The challenges of designing and implementing a doctoral student mentoring program. Innovative Higher Education, 37(3), 243-253.

Holley, K. A., \& Caldwell, M. L. (2012). The challenges of designing and implementing a doctoral student mentoring program. Innovative Higher Education, 37(3), 243-253.

Humphrey, R., \& Simpson, B. (2012). Negotiating a 'scary gap': Doctoral candidates,'writing up'qualitative data and the contemporary supervisory relationship. Journal of Education and Training Studies, 1(1), 1-10.

Humphreys, M. (2005). Getting personal: Reflexivity and autoethnographic vignettes. Qualitative Inquiry, $11(6), 840-860$.

Ives, G., \& Rowley, G. (2005). Supervisor selection or allocation and continuity of supervision: PhD students' progress and outcomes. Studies in Higher Education, 30(5), 535-555.

Jackson, J. (2015). Becoming interculturally competent: Theory to practice in international education. International Journal of Intercultural Relations, 48(Sept), 91-107.

Kearn, H., \& Gardiner, M. (2012). The seven secrets of highly successful research students. Adelaide: Flinders Press.

Kennedy, G. E., Judd, T. S., Churchward, A., Gray, K., \& Krause, K.-L. (2008). First year students' experiences with technology: Are they really digital natives. Australasian Journal of Educational Technology, 24(1), 108-122. 
Littlefield, C., Taddei, L., \& Radosh, M. (2015). Organic collaborative teams: The role of collaboration and peer to peer support for part-time doctoral completion. International Journal of Doctoral Studies, 10, 129-142. Retrieved from https://www.informingscience.org/Publications/2113

Mason, M. A., Goulden, M., \& Frasch, K. (2009). Why graduate students reject the fast track. Academe, 95(1), 11-16.

McClure, J. W. (2007). International graduates' cross-cultural adjustment: Experiences, coping strategies, and suggested programmatic responses. Teaching in Higher Education, 12(2), 199-217.

McCroskey, J. C., \& Richmond, V. P. (1990). Willingness to communicate: Differing cultural perspectives. Southern Journal of Communication, 56(1), 72-77.

Merga, M. (2015). Thesis by publication in education: An autoethnographic perspective for educational researchers. Issues in Educational Research, 25(3), 291-308.

Mezirow, J. (1978). Perspective transformation. Adult Education Quarterly, 28(2), 100-110.

Moxham, L., Dwyer, T., \& Reid-Searl, K. (2013). Articulating expectations for PhD candidature upon commencement: Ensuring supervisor/student 'best fit'. Journal of Higher Education Policy and Management, 35(4), 345-354.

Nestojko, J. F., Bui, D. C., Kornell, N., \& Bjork, E. L. (2014). Expecting to teach enhances learning and organization of knowledge in free recall of text passages. Memory \& Cognition, 42(7), 1038-1048.

Pearson, M., Cumming, J., Evans, T., Macauley, P., \& Ryland, K. (2011). How shall we know them? Capturing the diversity of difference in Australian doctoral candidates and their experiences. Studies in Higher Education, 36(5), 527-542.

Pearson, M., Cumming, J., Evans, T., Macauley, P., \& Ryland, K. (2011). How shall we know them? Capturing the diversity of difference in Australian doctoral candidates and their experiences. Studies in Higher Education, 36(5), 527-542.

Pyhalto, K., Toom, A., Stubb, J., \& Lonka, K. (2012). Challenges of becoming a scholar: A study of doctoral students of becoming a scholar. ISRN Education, 2012, 1-12. doi:10.5402/2012/934941

Ryan, R. M., \& Deci, E. L. (2000). Intrinsic and extrinsic motivations: Classic definitions and new directions. Contemporary Educational Psychology, 25(1), 54-67.

Service, R. W., \& Guess, A. K. (2015). Leadership advantage: A people person. Journal of Leadership, Accountability and Ethics, 12(4), 59.

Starr, L. J. (2010). The use of autoethnography in educational research: Locating who we are in what we do. CJNSE/RCJCÉ, 3(1).

Struthers, J. (2014). Analytic autoethnography: One story of the method. Theory and Method in Higher Education Research II, 183-202.

Thompson, N., \& Pascal, J. (2012). Developing critically reflective practice. Reflective Practice, 13(2), 311-325.

van de Schoot, R., Yerkes, M. A., Mouw, J. M., \& Sonneveld, H. (2013). What took them so long? Explaining $\mathrm{PhD}$ delays among doctoral candidates. PloS one, 8(7), 1-11.

Wall, S. (2008). Easier said than done: Writing an autoethnography. International Journal of Qualitative Methods, 7(1), 38-53.

Yates, L. (2010). Quality agendas and doctoral work. The tacit, the new agendas, the changing contexts. In P. Thomson \& M. Walker (Eds.), The Routledge doctoral student's companion (pp. 299-310). Abingdon, Oxon: Routledge. 


\section{Biographies}

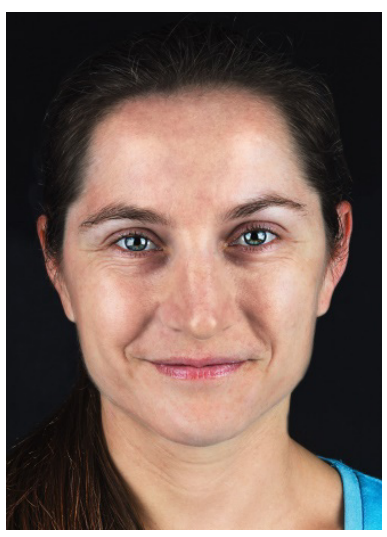

Sarah Booth is a doctoral candidate at Edith Cowan University and is currently working at the SOAR Centre in the Graduate Research School. After completing her Masters which focused on teaching Indigenous curriculum content she decided to take the topic further in a $\mathrm{PhD}$. She also conducts workshops on qualitative data software programs to both students and staff at universities around Australia.

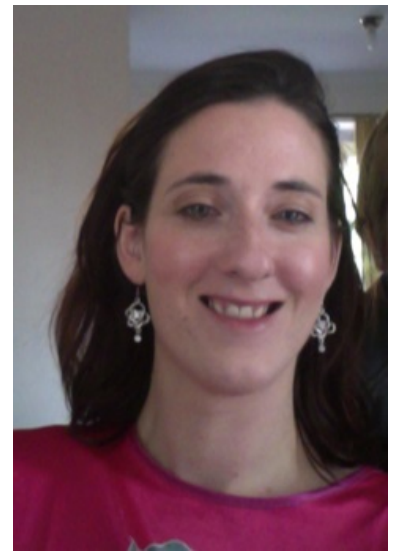

Dr Margaret Kristin Merga is a lecturer and early career researcher at Murdoch University who has taught English both in Australia and internationally. In 2012 she conducted the West Australian Study in Adolescent Book Reading, which examined adolescents' attitudes toward reading books for recreation, and the impact of social influences on adolescent engagement in the practice. Since then she has worked on a range of projects concerned with reading across the life span. She is also known for her research in health promotion and higher education.

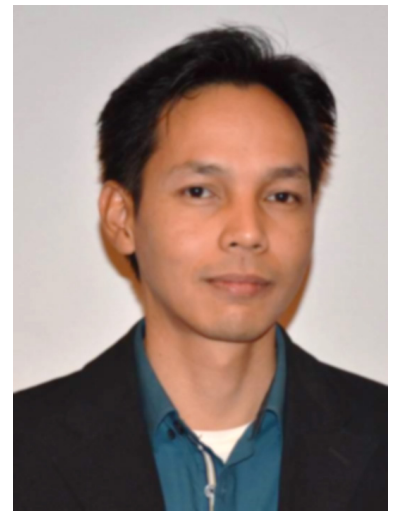

Dr Saiyidi Mat Roni completed his doctorate in accounting at Edith Cowan University before returning home to Malaysia where he works as a sought after lecturer at MARA University of Technology. He is also a research fellow at Accounting Research Institute in his home country. He still collaborates with ECU, regularly returning to conduct data analysis workshops to both staff and students. 